\title{
Intravascular lymphoma of the central nervous system: a rare subtype of a common disease
}

Linfoma intravascular do sistema nervoso central: um subtipo raro de uma condição comum

Ruann Melo de CARVALHO', Ana Paula Alves FONSECA', Igor Gomes PADILHA', Suellen Ka Gi MO²,

Flávia Fernandes Silva ZACCHI , Rita de Cássia Maciel PINCERATO', Carlos Sérgio CHIATTONE²

A 67-year-old man had a sudden onset of headache and aphasia, evolving to spontaneous improvement. A few months later, he developed dysarthria and left hemiparesis. Brain MRI showed several punctate lesions with perilesional edema, perivascular enhancement and restricted diffusion on DWI (Figure 1). PET-CT demonstrated mild uptake (Figure 1). Anatomopathological and immunohistochemical analysis were compatible with intravascular large B-cell lymphoma (IVL) (Figure 2). Treatment with R-CHOP and intrathecal methotrexate was established, with favorable response due to high tumor sensitivity ${ }^{1}$. Approximately half of IVL cases are diagnosed only after autopsy'2. The main differential diagnoses are vasculitis, neurosarcoidosis, and ischemic stroke ${ }^{3,4}$.

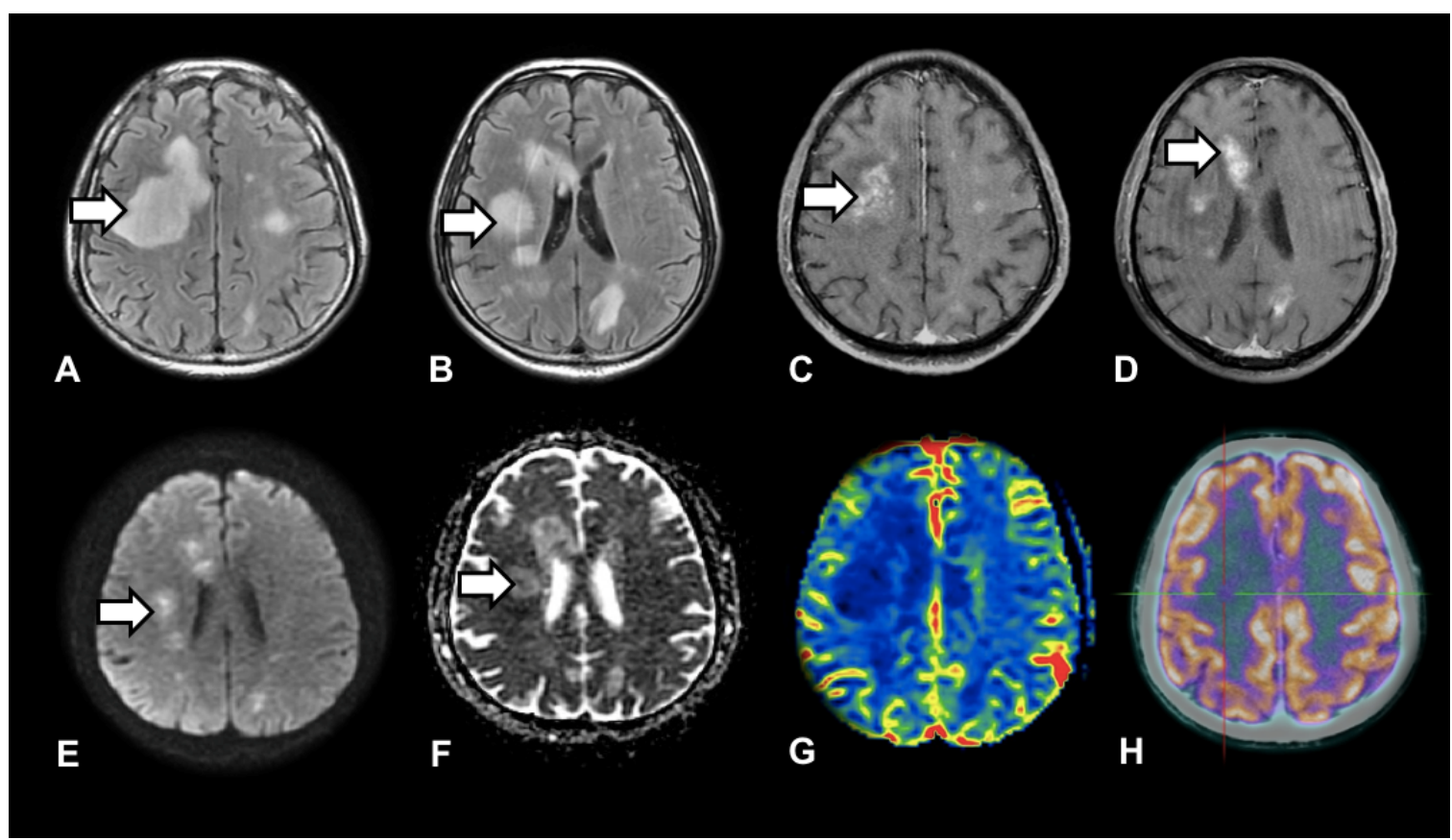

Figure 1. Magnetic resonance imaging findings of intravascular lymphoma. (A and B) Fluid-attenuated inversion recovery axial images showed diffuse multiple hyperintensities of the cerebral white matter. (C and D) The perivascular curvilinear enhancement on T1-weighted imaging with gadolinium expanded markedly. (E and F) Diffusion-weighted imaging and apparent diffusion coefficient map showed restricted diffusion. ( $\mathrm{G}$ and $\mathrm{H}$ ) Perfusion magnetic resonance imaging (relative cerebral blood volume) and positron emission tomography - computed tomography were practically unremarkable.

\footnotetext{
'Hospital Samaritano Higienópolis, UnitedHealth Group, Departamento de Neurorradiologia, São Paulo SP, Brazil.

${ }^{2}$ Hospital Samaritano Higienópolis, UnitedHealth Group, Departamento de Hematologia, São Paulo SP, Brazil.

${ }^{3}$ Grupo Fleury, Departamento de Anatomia Patológica, São Paulo SP, Brazil.

Ruann Melo de CARVALHO (DD https://orcid.org/0000-0002-6513-2069; Ana Paula Alves FONSECA (DD https://orcid.org/0000-0001-7112-7787; Igor Gomes PADILHA (D) https://orcid.org/0000-0002-7639-0397; Suellen Ka Gi MO (iD) https://orcid.org/0000-0002-4559-5859;

Flávia Fernandes Silva ZACCHI (D) https://orcid.org/0000-0002-2476-5994; Rita de Cássia Maciel PINCERATO (D) https://orcid.org/0000-0002-3672-6749; Carlos Sérgio CHIATTONE (D) https://orcid.org/0000-0002-4639-2578
}

Correspondence: Ruann Melo de Carvalho; E-mail: ruannmeloc@gmail.com

Conflict of interest: There is no conflict of interest to declare.

Authors' contributions: RMC, APAF, IGP, SKGM, FFSZ, RCMP, CSC: design or conceptualization of the study, analysis or interpretation of the data, drafting or revising the manuscript for intellectual content.

Received on September 12, 2020; Accepted on September 19, 2020. 

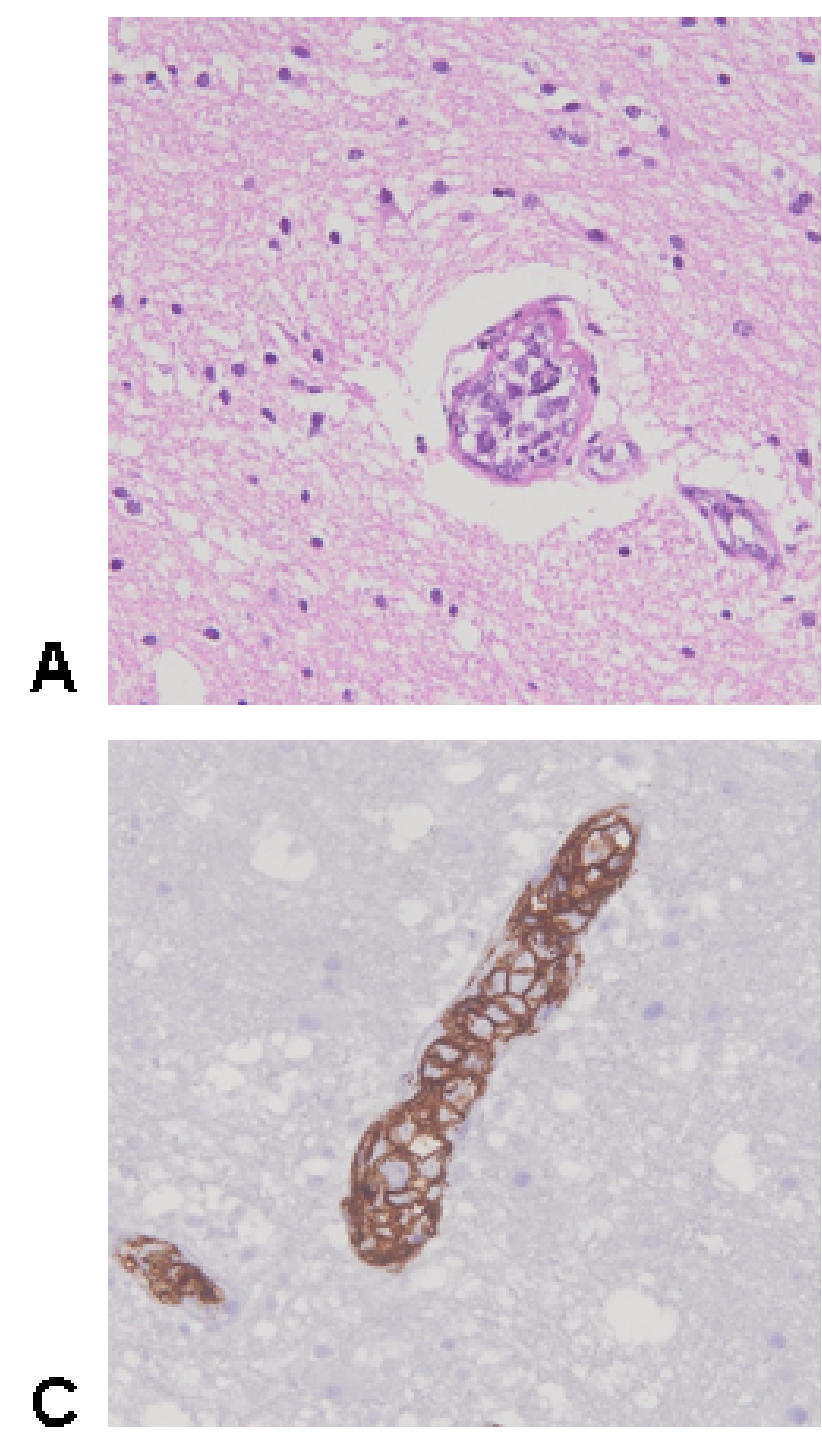
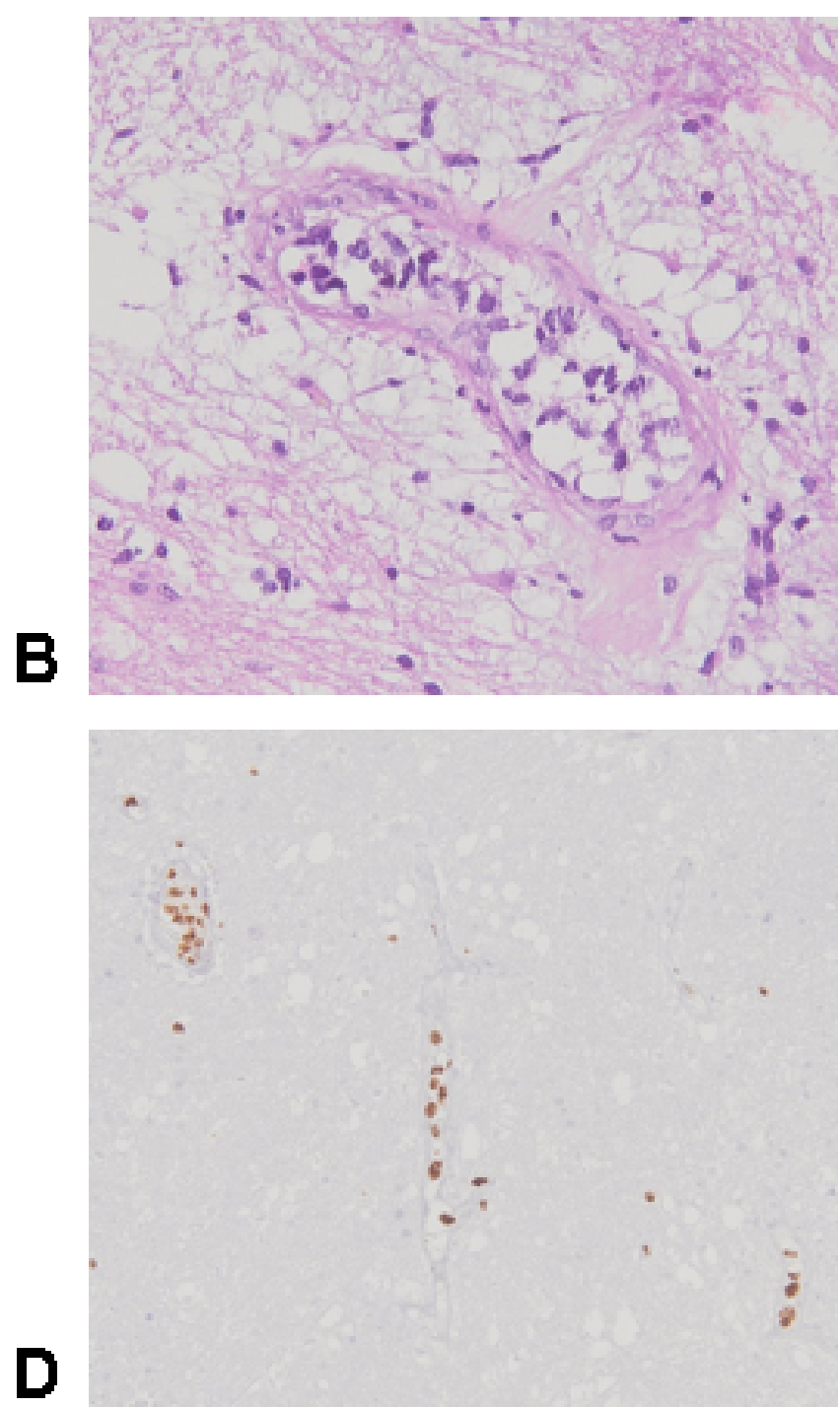

Figure 2. Brain pathology showing small blood vessels filled with lymphoma cells and perivascular reactive lymphocytes (A and B). Immuno his to chemical staining showing CD20+ (C and D).

\section{References}

1. Fonkem E, Lok E, Robison D, Gautam S, Wong ET. The natural history of intravascular lymphomatosis. Cancer Med. 2014 Jun;3(4):1010-24. wttps://doi.org/10.1002/cam4.269

2. Domizio P, Hall PA, Cotter F, Amiel S, Tucker J, Besser GM, et al. Angiotropic large cell lymphoma (ALCLL): morphological, immunohistochemical and genotypic studies with analysis of previous reports. Hematol Oncol. 1989 May-Jun;7(3):195-206. https://doi.org/10.1002/hon.2900070303
3. Williams RL, Meltzer CC, Smirniotopoulos JG, Fukui MB, Inman M. Cerebral MR imaging in intravascular lymphomatosis. AJNR Am J Neuroradiol. 1998 Mar;19(3):427-31.

4. Baehring JM, Henchcliffe C, Ledezma CJ, Fulbright R, Hochberg FH. Intravascular lymphoma: magnetic resonance imaging correlates of disease dynamics within the central nervous system. J Neurol Neurosurg Psychiatr. 2005 Apr;76(4):540-4. https://doi.org/10.1136/ jnnp.2003.033662 